\title{
Distinguishing between coronal cloud prominences and channel prominences and their associations with solar and stellar flares
}

\author{
Sara F. Martin ${ }^{1}$, Oddbjorn Engvold ${ }^{2}$, Yong Lin $^{3}$ and \\ Jacqueline Alves da Silva ${ }^{4}$ \\ ${ }^{1}$ Helio Research, 5212 Maryland Ave., La Crescenta, CA 91214, USA \\ email: sara@helioresearch.org \\ ${ }^{2}$ Institute of Theoretical Astrophysics, University of Oslo, \\ P.O. Box 1029, Blindern, 0315 Oslo, Norway \\ email: oddbjorn.engvold@astro.uio.no \\ ${ }^{3}$ Geodetic Institute, Norwegian Mapping Authority, \\ Kartverksveien 21, 3511 Honefoss, Norway \\ email: angeline_lin@hotmail.com \\ ${ }^{4}$ Florida Institute of Technology, Melbourne, FL, USA \\ email: jalvesdasilv2015@my.fit.edu
}

\begin{abstract}
To better understand the differences between coronal cloud prominences and channel prominences, we systematically identified and analyzed coronal cloud prominences recorded in SDO/AIA images at $304 \AA$ from 2010 May 20 through 2012 April 28. For the 225 cases identified, their numbers vary directly with the sunspot number. Their durations are typically less than 3 days. Their most frequent maximum height is $90,000+$ and $-10,000 \mathrm{~km}$. We offer our hypothesis that many coronal cloud prominences originate from some of the mass of previously erupted filaments ejected high out of their filament channels; subsequently part of this mass falls and collects in leaky magnetic troughs among coronal magnetic fields which constrain the leaked mass to slowly drain downward along curved trajectories where it appears as coronal rain. Currently there is inadequate evidence for a convincing correspondence between either coronal cloud prominences or channel prominences with stellar prominences detected to date.
\end{abstract}

Keywords. prominences, filaments, corona

\section{Introduction}

Both coronal cloud prominences and channel prominences have been observed since the early history of solar astronomy (Secchi 1877). However, arriving at solid evidence, that any categories of long-lived prominences are truly magnetically different from each other, has been long and slow. Examples of coronal clouds are illustrated in the books on solar prominences (Tandberg-Hanssen 1974a, 1995, Vial \& Engvold 2015) and can be found in archival movies such as those of the High Altitude Observatory and the U. S. National Solar Observatory recorded at Sacramento Peak. Papers devoted exclusively to coronal cloud prominences are relatively rare (Leroy 1972, Allen et al. 1998, Liu et al. $2012,2014)$ as are papers about coronal rain specifically from coronal cloud prominences (Leroy 1972) and not associated with sunspots (Tandberg-Hanssen 1974b).

Another category of coronal rain, associated within flare loops or flare-like coronal loops over active regions Tandberg-Hanssen (1974c), is associated with the recycling of mass from the chromosphere (Brosier 2003, Muller et al. 2004, Anatolin and Rouppe van der Voort 2012, and Antolin et al. 2015). This latter category of coronal rain does 
not have a clear association with coronal cloud prominences. Rain from coronal cloud prominences is usually more irregular and coarse. Henceforth, this paper refers only coronal rain identified with coronal cloud prominences. Coronal cloud prominences begin at apparently isolated locations in the corona where mass evidently accumulates from unknown sources and subsequently drains to the chromosphere as coronal rain often flowing downward along thin arc-shaped trajectories. Sometimes multiples arcs emanate from a single cloud; this resulted in a fraction of them being informally called "spiders" (Allen et al. 1998).

Most of the remaining hundreds of papers on prominences in the literature describe long-lived ones that are very different from coronal cloud prominences in their brightness, structure, heights, mass motions, and environmental structures. Typically, they are long, low and well connected to the chromosphere either fully along their spines or at discrete structures extending from a spine called "barbs." To emphasize evidence that these prominences are magnetically different from coronal cloud prominences, Martin (2015) used the contrasting term "channel" prominences; this term calls attention to the findings described in many papers of the close relationship of and dependence of these prominences on the presence of an external magnetic environment called a "channel" whose central field is parallel with the prominence spine (Gaizauskas et al. 1998, 2001).

\section{Typical Coronal Cloud Prominences in $\mathbf{H} \alpha$}

Examples of two typical forms of coronal cloud prominences are illustrated in the second and third rows of images in Figure 1. They were recorded at Helio Research on 2004 Sep. 16 and 17 at adjacent locations on the west limb. The cloud parts of the prominences are the concentrations of mass that appear at relatively fixed locations in the corona. The time series of images, from which the samples in Figure 1 were taken, reveal mass continuously entering the clouds from above and leaving the bottoms of the clouds. The coronal rain from the clouds often consisted of successive flows along the same arcs for intervals of hours, sometimes beginning before and continuing after a related cloud is seen.

In the second row of Figure 1, the clouds and rain are relatively faint and only hint of the presence of arcs of several sizes. However, the upper part of the middle image at 16:46 in the third row reveals a clear arc of coronal rain that has continued into Sep 17. Typically, rain begins from the cloud or the top of an initially invisible arc and usually flows downward either along one half or both halves of the arc. There are only rare exceptions when a small upward component has been seen before the downward flows.

The high clouds of the coronal cloud prominence on Sep. 16 were not seen at all during on the next observing day at Helio Research. However, the new, larger, brighter coronal cloud prominence in the third row was visible at the beginning of the next observing day. The time series on Sep. 17 shows two tiers of clouds from which the rain appears to fall nearly vertically. When rain appears to fall nearly vertically, an open question is whether it is really flowing along an arc viewed from one end rather than from a perspective broadside to an arc. Flows downward along arcs seen from one end would appear to trace nearly vertical downward trajectories, consistent with the rain from this brighter and more extensive coronal cloud prominence on Sep. 17. Many more observations and studies are needed to learn if this supposition that differences in orientation relative to the observer account for at least some of the apparent variations among coronal cloud prominences and the paths traced by their coronal rain. 


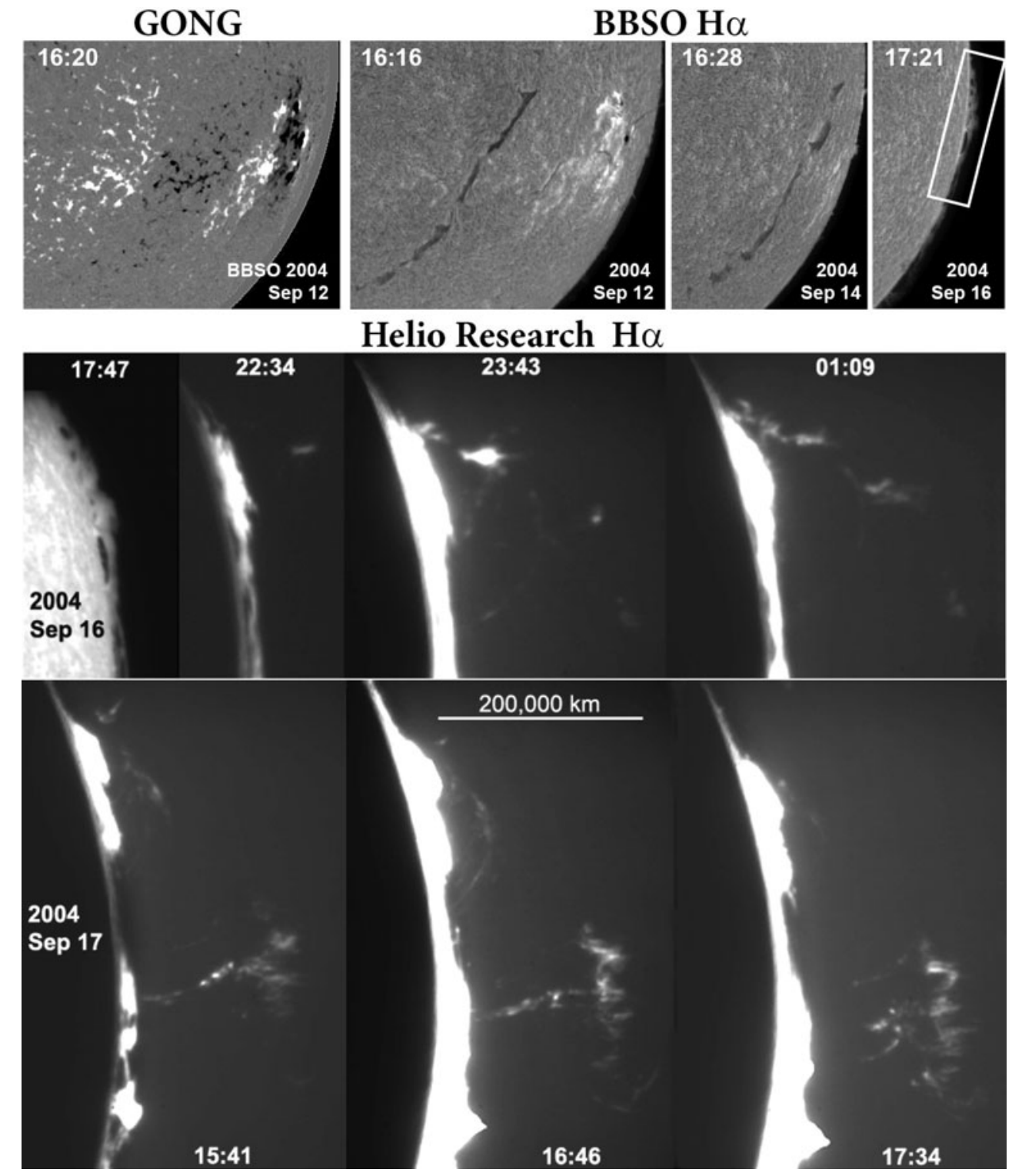

Figure 1. The second, third and fourth images in the first row show a typical long and low, $\mathrm{H} \alpha$ channel prominence approaching the limb due to solar rotation. The magnetogram in the first image reveals the location of the channel prominence along a boundary between positive (white) and negative (black) polarity fields. The white rectangle on the image at 17:21 shows the relative orientation of the Helio Research images in the next two rows which illustrate faint coronal cloud prominences up to the height of 200,000 km. Mass continuously enters these dynamic cloud prominences from above and drains from them as coronal rain. There is an absence of evidence of any relationship between the coronal cloud prominences and the channel prominence close to the limb. The coronal cloud prominences could be related to coronal fields around or above the bright active region complex seen on Sep 12 which would have been carried by solar rotation about 26 heliographic degrees behind the limb by the time the coronal cloud prominences were observed. 


\section{A Typical Channel Prominence in $\mathbf{H} \alpha$}

The long-lived prominence, along and close to the solar limb in the second and third rows in Figure 1, is a channel prominence. In exposures long enough to reveal the coronal cloud prominence, this channel prominence is severely overexposed. The earlier images in the first row show this channel prominence on preceding days in projection against the chromospheric disk as seen in sections of full disk $\mathrm{H} \alpha$ images from the Big Bear Solar Observatory.

When the channel prominence reaches the limb, in the long exposures from 22:34 onward, it appears to be below the coronal cloud prominence. However, to date, we have only negative evidence that coronal cloud prominences are suspended directly above channel prominences or their channels. Therefore, we suggest these coronal cloud prominences are most likely to be related to coronal magnetic fields beyond the limb, possibly to fields related to the bright complex of active regions seen at 16:16 on Sep 12 in Figure 1.

A magnetogram corresponding to the $\mathrm{H} \alpha$ image on Sep 12 is presented in the first image in the first row. The channel prominence lies along the boundary between the positive (white) and negative (black) polarity fields, from the upper middle to the lower left side of the magnetogram. This channel prominence can also be classed as a quiescent prominence because it is distant from any major active region. However, active region, intermediate and quiescent prominences all fall under the classification of channel prominences because the magnetic field configuration of the channels in which they occur are all similar, as illustrated in Martin (2015).

Another principal feature of channel prominences, illustrated in the first row of Figure 1 , is their visibility as bright structures in the solar corona beyond the limb in $\mathrm{H} \alpha$ but as dark features against the solar chromosphere. They are dark where their mass has sufficient density to scatter light from the chromosphere (or background corona) out of the observer's line-of-sight. When viewed in projection against the disk, channel prominences are commonly called "filaments." Because coronal cloud prominences have not been detected against the solar chromosphere, the term "filaments" is synonymous with the term "channel prominences".

The largest, identifiable, structural components of a channel prominence or filament are its spine and barbs. As seen in the filament in the first row of Figure 1, the spine of a filament is the mass along its main axis. Barb is the name for the section of a thread or group of threads of filament mass which extend away from the spine to the chromosphere on each side of the spine. Spines and barbs cannot be separate structures; when spatially resolved, the threads of the barbs are contiguous with threads of the spine. The terms, spine and barb, are simply useful for identifying segments of filaments (Martin 1998).

Although not shown herein, the basic building blocks of both filament spines and barbs are fine, dynamic threads aligned with the local magnetic field (Engvold 1998, 2004, Lin et al. 2003, 2008, Lin 2004). The mass flows that define the threads are continuous between the spine and barbs. However, the mass flows, in their steady state, are in opposing directions along interleaved threads throughout the spine and barbs, a property known as "counterstreaming" (Zirker et al. 1998), a property not shared by coronal cloud prominences. Through counterstreaming, the mass of channel prominences and filaments can be traced or deduced to come directly from the chromosphere or photosphere. However, even when the first appearance of prominence threads is seen as a condensation, such threads can still originate from mass ejected from the chromosphere or photosphere but be initially invisible due to having a higher temperature and/or lower density; with subsequent low density ejections, the overall density can increase and the temperature drop 
resulting in the mass becoming first visible at a location different than its source (Karpen 2015).

In contrast, the mass of coronal cloud prominences cannot be traced directly to these layers of the solar atmosphere. However, for channel prominences, due to some coherency in the mass motion in groups of adjacent threads, counterstreaming and streaming is readily detectable in time-lapse series of images observed at medium resolution. We emphasize that prominences seen at medium spatial resolution are adequate to distinguish between coronal cloud prominences and channel prominences.

\section{Prominences Detected from Satellite Experiments in Space}

In $304 \AA$ images from SOHO, SDO and STEREO A and B satellites, coronal cloud prominences and channel prominences can be distinguished from each other. Lin (2000) and Wang (2001) have shown that both spines and barbs are seen in $304 \AA$ as well as in $\mathrm{H} \alpha$ and Liu et al. $(2012,2014)$ have analyzed specific examples of coronal cloud prominences. In images at $304 \AA$, fainter prominences and fainter parts of prominences can be observed more readily than in $\mathrm{H} \alpha$.

Lin (2000) has shown that the lower parts of prominences are more structurally clear in $\mathrm{H} \alpha$ while higher, fainter parts are revealed in $304 \AA$ that are not seen in $\mathrm{H} \alpha$. From these previous studies and viewing $304 \AA$ images available over the internet, we learned to expect to find more coronal cloud prominences in the $304 \AA$ images. Additionally, the nearly continuous 24 hour per day images from the experiments on board the SOHO and SDO satellites provided the possibility of collecting a sufficient sample of coronal cloud prominences for an initial statistical study of their properties. No adequate statistical sample had been collected previously because coronal cloud prominences were relatively rare in $\mathrm{H} \alpha$. This is because few prominences above the limb are detectable in $\mathrm{H} \alpha$ images with exposures suitable for chromospheric structures. Most prominences up to the time of the launch of SOHO had been detected in $\mathrm{H} \alpha$ images in which the disk had been intentionally overexposed such as in the full disk prominence monitor images from the Mauna Loa observing station of the High Altitude Observatory. However, the degree of overexposing still favors channel prominences over the fainter coronal cloud prominences. Therefore, filaments or channel prominences have been much more thoroughly recorded and studied than coronal cloud prominences.

\section{A Statistical Study of Coronal Cloud Prominences}

To learn more about coronal cloud prominences, we initiated a statistical study of them for an approximate 2 year interval from 2010 May 20 to 2012 April 28 using $304 \AA$ images from the Solar Dynamics Observatory (SDO). We used the Atmospheric Imaging Assembly and Heliospheric Magnetic Imager (AIA/HMI) browser on the SDO web pages to make temporary movies in $304 \AA$ in which we could recognize the appearance and dynamics of coronal cloud prominences. We settled on the following criteria that a coronal cloud prominence should exhibit for inclusion in our statistics:

(a) a nearly fixed site where mass appeared to collect and from which mass flowed downward

(b) a duration of visible mass for 4 or more hours

(c) in the cases of clustering of downward flowing sites of coronal rain or clouds, events were considered to be independent if separated by approximately 10 degrees or more at the point on the limb vertically below the mass source. 
(d) repetitions of separate strands of coronal cloud prominence mass at approximately the same location were counted as belonging to the same event if these separate strands occurred within a few degrees of the same coronal location and less than half a day apart in time.

These criteria include both coronal rain without an apparent cloud as well as coronal rain coming from a coronal cloud. Whether rain occurs with or without a cloud might depend on the magnetic topology, which either could support a cloud from which mass drains as rain, or alternatively, could allow mass to drain as quickly as it appears to condense (Engvold 2015). The number of coronal cloud prominences found to fit our criteria was 225 in the interval from 2010 May 20 through 2012 April 28. This result demonstrated that coronal cloud prominences are not rare as we had supposed from the relative paucity of their numbers reported in the literature as well as their numbers casually seen while personally reviewing long duration $\mathrm{H} \alpha$ data sets.

The distribution of the coronal clouds over time by Carrington rotation is shown in Figure 2 a for comparison with the distribution of sunspot numbers over the same interval in Figure 2b. The number of coronal clouds has three peaks corresponding well with the 3 peaks in the sunspot number.

Figure 2c is a distribution of erupting prominences from the paper by McCauley et al. (2015); this distribution is of interest because we wish to test our hypothesis that coronal cloud prominences might acquire their downward flowing mass from some of the mass of erupting filaments that was previously ejected into the high, outer corona but did not reach escape velocity. Apparently, only a small fraction of filaments or parts of filaments reach escape velocity and are detectable with CMEs in space (Ruzmaikin et al. 2003). However, the mass of many filaments fall back into the channels from which they come. Therefore, not all eruptives are expected to contribute to the population of coronal cloud prominences. Many filaments from within or near active regions erupt energetically, and spew their mass over a wide volume of space outside of their channels and their associated active regions. This is illustrated in Figure 3 and in the paper of van Driel-Geztelyi (see contribution of van Driel-Gezstelyi this volume). Such eruptions are the candidates for providing mass for coronal cloud prominences up to a day after their eruption because some of their mass can attain heights of many solar radii before slowing, stopping, and falling back to the Sun (Gopalswamy 2015).

The second row of images in Figure 3 shows an example of a coronal cloud prominence approximately 14 and 20 hours respectively (frames 00:38 UT and 06:23 UT) after the mass that previously fell during the main part of the event in the lower atmosphere seen at 07:41 UT and 10:00 UT in the first row of Figure 3. Due to the limited field of view of the $304 \AA$ images from SOHO, SDO and STEREO, we have limited knowledge of the full range of heights that prominence mass attains during CMEs.

Fig $2 \mathrm{~d}$ is the distribution of heights in increments of $10,000 \mathrm{~km}$. It is a relatively symmetric distribution with a modal height of $90,000 \mathrm{~km}$. For comparison, we show in Fig. 2e, a graph from Filippov and Den (2001) to learn if there is a critical height for quiescent filaments above which they invariably erupt. They found that such a critical height exists but it is dependent on a number of parameters. However, with one exception, the filaments in their study, which survived passage across the west limb, were not higher than $65,000 \mathrm{~km}$. In contrast, about two thirds of the all the coronal cloud prominences in our study had maximum heights greater than $65,000 \mathrm{~km}$. Therefore, if one desired to use the critical height for anticipating or forecasting the eruption of channel prominences above the limb, it would be important to be able to detect and clearly distinguish them from coronal cloud prominences in order to eliminate coronal cloud prominences from consideration. 


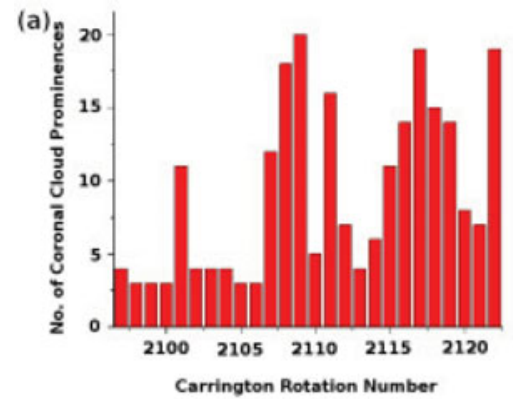

(b)

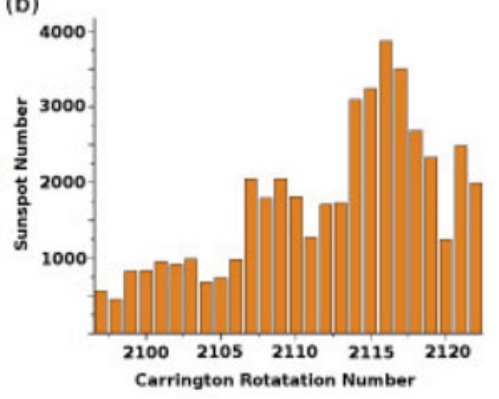

(c)

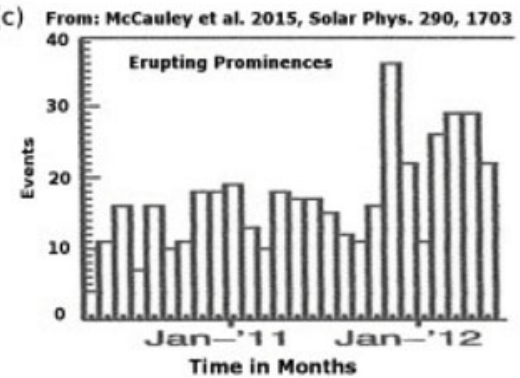

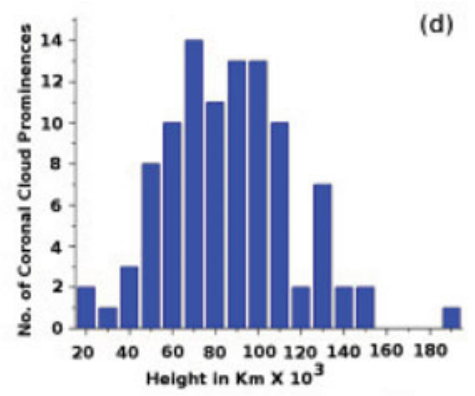

(e)
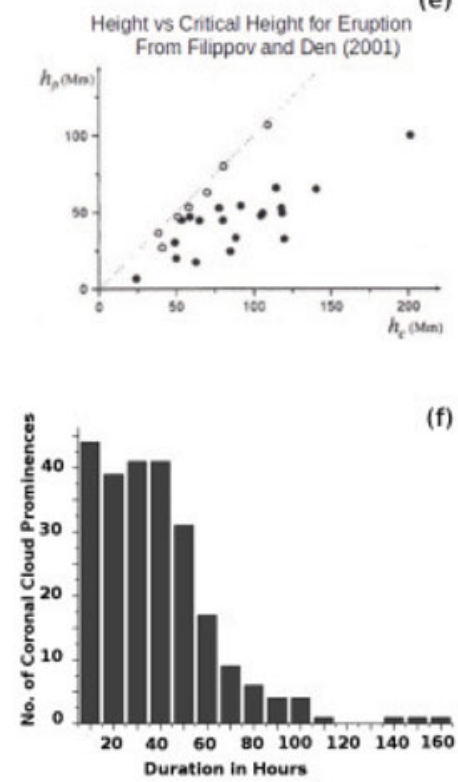

Figure 2. (a) The distribution of 225 Coronal Cloud Prominences in $304 \mathrm{~A}$ data from SDO/AIA from 2010 May 20 - 2012 April 28 compared with (b) the Sunspot Number and (c) the distribution of erupting prominences during the same interval but given by month from McCauley et al. (2015). (d) The distribution of the maximum heights for a sample of 100 of the coronal cloud prominences can be compared with (f) a graph from Filippov and Den (2001) showing a diagonal representing the critical height for the erupting prominences; those that erupted are represented by open circles and those that crossed the west limb without erupting are represented by filled circles. (f) The distribution of the durations of the coronal cloud prominences for the same sample as in the distribution over time in Figure 2(a).

Fig $2 \mathrm{f}$ is the distribution of the durations of coronal cloud prominences in increments of 10 hours. The majority have durations less than 3 days (72 hours). Nearly as many last 50 hours as 10 hours. After 2 days (48 hrs.) the durations drop off steeply with increasing time. The few, that appear to be very long-lived, might be successive events rather than single events; our criteria for single events still allows for sporadic recurrence of mass at or close to the same coronal site.

\section{On Possible Stellar Associations}

Stellar prominences are found on some of the same types of stars that have stellar flares. Also the light curves and solar flares and stellar flares have similar intensity profiles over 


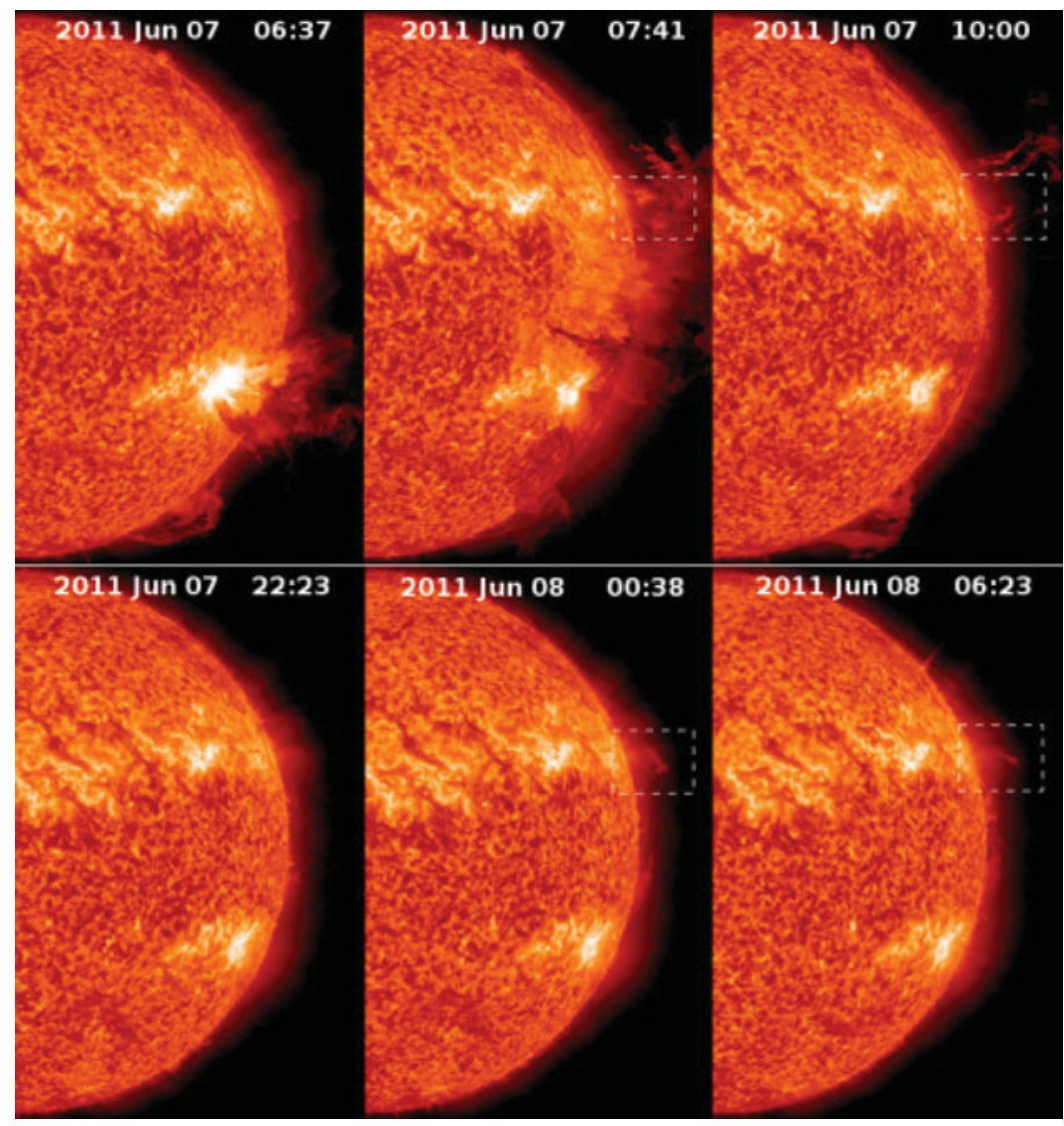

Figure 3. A possible source of mass for coronal cloud prominences is the mass from erupting prominences especially the energetic, small, dense ones from active regions because their mass is thrown far out of their channels of origin as seen in the first row of images. Most of the time, erupting prominence mass is seen for only a short time before it is ejected out of the field of view. However, it has been shown that erupting prominence mass can attain heights of many solar radii before falling back to the Sun up to a day after the original event. Limitations in the fields-of-view of the SDO/AIA and other imagers on satellites in space, currently prevent our knowing what percentage of coronal cloud prominences and their coronal rain are due to prior erupting prominences and what percentage might be due to other mechanisms.

time, with both having a sharper rise than decay although flares in solar-like and later type stars often reach energies that are orders of magnitude higher than solar flares (Pettersen 1989).

Furthermore, stars with spots are among those that also have flares. If the analogies continue, it would be reasonable to expect both channels prominence and coronal cloud prominences on other stars. However, the only technique yet proven to work for finding prominences on other stars is only applicable to those with rapid rotation which strongly affects the hydrostatic conditions in these stars. Stellar prominences extend to heights of several stellar radii. Little is known about how they form and how their existence is terminated. Erupting stellar prominences have not been identified (Jardin et al. 1998, Hussain 2013). These differences are enough to suspect that channel prominences do not correspond to stellar prominences. On the other hand, rapid rotation changes the whole atmosphere of a star so much that channel prominences cannot be ruled as candidates for the elevated prominences observed. 
Coronal cloud prominences seem like much better candidates but their densities are far below that required to detect stellar prominences. Stellar prominences are detectable due to their Doppler shifts that locate them relative to their host stars. Much more needs to be known about stellar prominences especially how they acquire and lose mass to know whether they could be analogous to coronal cloud prominences on the Sun. More information about the properties of coronal cloud prominences would also be beneficial to addressing these questions in the future.

\section{Acknowlegments}

We thank the Brazilian Scientific Mobility Program (BSMP) for the Internship of J. Alves da Silva at Helio Research which enabled her participation in this research and the Florida Institute of Technology as her primary hosting institution during her scholarship supported by the BSMP.

\section{References}

Allen, U. A., Bagenal, F., \& Hundhausen, A. 1998, in: D. Webb, D. Rust, and B. Schmieder (eds.), New Perspectives on Solar Prominences, Proc. IAU Colloquium No. 167, (San Francisco: ASP Conf. Series), 150, 290

Antolin, P. \& Rouppe van der Voort, L. 2012, ApJ, 745, 152

Antolin, P., Vissers, G., Pereira, T. M. D., Rouppe van der Voort, L.,\& and Scullion, E. 2015, ApJ, 806, 81

Brosius, Jeffrey W. 2003, ApJ, 586, 1417

Engvold, O. 1998, in: D. Webb, D. Rust, and B. Schmieder (eds.), New Perspectives on Solar Prominences, Proc. IAU Colloquium 167, (San Francisco: ASP Conf. Series), 150, 23

Engvold, O. 2004, in: A.V. Stepanov, E.E. Benevolenskaya, \& A.G. Kosovichev (eds.), MultiWavelength Investigations of Solar Activity, Proc. IAU Symposium 223 (Cambridge, UK: Cambridge University Press), p. 187

Engvold, O. 2015, J.-C. Vial \& O. Engvold (eds.), Solar Prominences, (Astrophysics and Space Science Library), 415, 31

Filippov, B. P. \& Den, O. G. 2001, J. Geophys. Res., 106, 25177

Gaizauskas, V., Zirker, J. B., Sweetland, C., \& Kovacs, A. 1977, ApJ, 479, 448

Gaizauskas, V., Mackay, D. H., \& Harvey, K. L. 2001, ApJ, 558, 888

Gopalswamy, N. 2015, in: J.-C. Vial \& O. Engvold (eds.), Solar Prominences,(Astrophysics and Space Science Library), 415, 381

Hussain, G. A. J. 2014, B. Schmieder, J.-M. Malherbe \& S.T. Wu (eds.), Nature of Prominences and their Role in Space Weather, Proc. IAU Symposium 300 (Cambridge: Cambridge University Press ), p. 309

Jardine, M., Barnes, Y., Unruh, Y., \& Cameron, A. C. 1998, in: D. Webb, D. Rust, and B. Schmieder (eds.), New Perspectives on Solar prominences, Proc. IAU Colloquium 167, (San Francisco: ASP Conf. Series), 150, 235

Karpen, J. 2015, in: J.-C. Vial \& O. Engvold (eds.), Solar Prominences, (Astrophysics and Space Science Library), 415, 237

LeRoy, J.-L. 1972, Solar Phys., 25, 413L

Lin, Y. 2000, MA Thesis, Institute of Theoretical Astrophysics, University of Oslo

Lin, Y., Engvold O. \& Wiik, J. E. 2003, Solar Phys., 216, 109

Lin, Y. 2004, PhD Thesis, Institute of Theoretical Astrophysics, University of Oslo

Lin, Y., Engvold, O., Rouppe van der Voort, L., Wiik, J. E., \& Berger, T. E. 2005, Solar Phys., 226, 239

Lin, Y., Martin, S. F., \& Engvold, O. 2008, A.G.U. Spring Meeting, ABS No. SH23A-05

Liu, W., Berger, T. E., \& Low, B. C. 2012, Ap. Lett., 745, L21

Liu, W., Berger, T. E., \& and Low, B. C. 2014, in: B. Schmieder, J.-M. Malherbe \& S. T. 
$\mathrm{Wu}$ (eds.), Nature of Prominences and their role in Space Weather Proc. IAU Symposium (Cambridge: Cambridge University Press ), p. 441

Martin, S. F. 1998, Solar Phys., 182, 107

Martin, S. F. 2015, in: J.-C. Vial \& O. Engvold (eds), Solar Prominences, (Astrophysics and Space Science Library) Chap. 9

McCauley, P. I., Su, Y. N., Schanche, N., Evans, K. E., Su, C., McKillop, S., \& Reeves, K. K. 2015, Solar Phys., 290, 1703

Muller, D. A. N., de Groof, A., Hansteen, V. H., \& Peter, H. 2004, in: R.W. Walsh, J. Ireland, D. Danesy, B. Fleck (eds.), Coronal Heating, Proc. of the SOHO 15 Workshop ( Paris: European Space Agency), p. 291

Pettersen, B. R. 1989, Solar Physics, 121, 299

Ruzmaikin, A., Martin, S., \& Hu, Q. 2003, J. Geophys. Res., (Space Physics), 108, p. 13-1

Secchi, P. A. 1877, Le Soleil, (image reproduced in frontispiece of The Nature of Solar Prominences by E. Tandberg-Hanssen), p. 0

Tandberg-Hanssen, E. 1974a, Solar Prominences, (Dordrecht: D. Reidel Pub. Co.) p. 0 (frontispiece, equatorial prominence above east limb)

Tandberg-Hanssen, E. 1974b, Solar Prominences, (Dordrecht: D. Reidel Pub. Co.) p. 8

Tandberg-Hanssen, E. 1974c, Solar Prominences, (Dordrecht: D. Reidel Pub. Co.) p. 10

Tandberg-Hanssen, E. 1995, The Nature of Solar Prominences, (Dordrecht: D. Reidel Pub. Co.) p. 0 (frontispiece, equatorial prominence above east limb)

Vial J.-C. and Engvold, O. 2015, in: Vial J.-C. and Engvold, O. (eds.), Solar Prominences, (New York: Springer)

Wang, Y.-M. 2001, ApJ, 560, 456

Zirker, J. B., Engvold, O., \& Martin, S. F. 1998, Nature, 396, 440 